\title{
Pulsatility Index as a Novel Parameter for Perfusion in Mouse Model of Hindlimb Ischemia
}

\author{
Hairong Xu $\mathrm{Xu}^{\mathrm{a}}$ Shan Lu ${ }^{\mathrm{b}}$ Lexi Ding ${ }^{c}$ Liangkun Lyu ${ }^{\mathrm{d}}$ Zejun Ma ${ }^{\mathrm{e}}$ Qiulun $\mathrm{Lu}^{f}$ \\ ${ }^{a} C l i n i c$ Medical Collage, Jiangsu Key Lab Integrated Tradit Chinese \& Weste, Jiangsu Coinnovat Ctr \\ Prevent \& Control Important, Jiangsu Key Lab Zoonosis, School of Medicine, Yangzhou University, \\ Yangzhou, China, ${ }^{b}$ Department of Pharmacology, University of California, Davis, USA, 'Department of \\ Ophthalmology, Xiangya Hospital, Central South University, Changsha, d3PM-Wuhan, Wuhan, eKey \\ Laboratory of Hormones and Development (Ministry of Health), Tianjin Key Laboratory of Metabolic \\ Diseases, Tianjin Metabolic Diseases Hospital \& Tianjin Institute of Endocrinology, Tianjin Medical

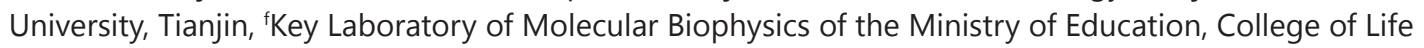 \\ Science and Technology and Center for Human Genome Research, Huazhong University of Science and \\ Technology, Wuhan, China
}

\section{Key Words}

Pulsatility index • Hindlimb ischemia • Perfusion • Blood flow

\begin{abstract}
Background/Aims: In clinical settings, the pulsatility index (PI) has become a widely used tool for monitoring obstetrics or other vascular diseases. It is based on the maximum Doppler shift waveform derived from ultrasonography. However, it remains unclear whether the PI levels are correctly predicted from the perfusion in mouse model of hindlimb ischemia. Methods: To explore the relationship between PI and perfusion, we generated a unilateral hindlimb ischemia model in 8-week-old C57BL/6 male mice by ligation of the right common iliac artery and femoral artery. These mice were monitored with laser Doppler perfusion imaging (LDPI) and an ultrasound system (Vevo2100). Vessel densities in ischemic skeletal muscles were measured with vWF staining, which functions as a marker for endothelial cells. In order to further verify PI evaluation in other conditions, we performed therapeutic experiments using hindlimb ischemic mouse with PBS or FGF2 treatment. Results: In the mouse model of hindlimb ischemia, the PI levels were continuously elevated and were accompanied by an increased ratio of perfusion to blood flow. 1 and 4 weeks after ischemia, the densities of VWF staining were correlated with PI values. Moreover, the PI index exactly reflected the perfusion in hindlimb ischemic mice after FGF2 treatment, while it indicated the condition of angiogenesis after therapeutic treatment based on the association between PI values and the number of vWF-positive stained cells in muscles. Conclusion: This study confirms the utility
\end{abstract}

Hairong Xu and Shan Lu contributed equally to this work.

\begin{tabular}{ll}
\hline Qiulun Lu PhD & Key Laboratory of Molecular Biophysics of the Ministry of Education; College of Life Science and Tech. \\
and Center for Human Genome Research, Huazhong Univ. of Science and Tech., Wuhan, (China) \\
E-Mail aaron.ql.lu@gmail.com
\end{tabular}




\section{Cellular Physiology Cell Physiol Biochem 2018;48:2114-2122

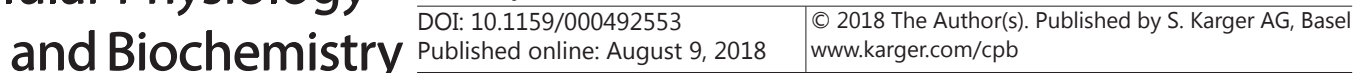 \\ Xu et al.: Pulsatility Index for Perfusion Evaluation}

of a noninvasive and reproducible ultrasound index for a rapid evaluation of perfusion and blood recovery after hindlimb ischemia in vivo. PI, as one stable and comparable parameter, is correlated with angiogenesis in hindlimb ischemic mouse. Moreover, PI can exactly reflect perfusion and angiogenesis in therapeutic hindlimb ischemic mouse models. This study suggested that PI can serve as a novel index for relatively reproducible and repeatable blood flow recovery in the evaluation of emerging ischemic therapies and disease development in mouse models of hindlimb ischemia.

\section{Introduction}

The prevalence of peripheral arterial disease (PAD) has been increasing across the aging population and results in progressive narrowing and occlusion of the peripheral arteries [1]. Approximately $12 \%$ of adults are diagnosed with this disease [2]. In Germany, PAD prevalence in women and men aged over 65 years is $17 \%$ and $20 \%$, respectively [3]. In some severe cases, PAD significantly affects the quality of life, resulting in high morbidity and mortality [4]. Patients with both diabetes and PAD are at higher risk of low extremity amputation than those without diabetes [5]. Among those suffering from both diseases, a quarter of patients affected with critical limb ischemia died each year [6]. In patients with PAD alone, susceptibility of coronary artery disease (CAD) ranges from $46 \%$ to $71 \%[7,8]$. Unfortunately, until now, there is no cure for this disease.

The assessment of blood flow recovery in small animals is critical for phenotyping genetic mouse models, evaluating the efficacy of novel vascular disease therapies, and investigating the safety of agents with potential toxic side effects $[9,10]$. Conventional noninvasive in vivo methods for assessing blood flow recovery are used for physiological investigations but are not reproducible, which prevents comparisons. The current imaging techniques, such as laser Doppler imaging [11], contrast-enhanced power Doppler ultrasound imaging, pulsedwave velocity ultrasound [12, 13], magnetic resonance imaging (MRI) [14], computed tomography (CT) [15], single-photon emission computed tomography (SPECT) [16], and positron emission tomography (PET) [17], are hampered due to compromises between spatial and temporal resolution, penetration, and sensitivity. These methods might be unsuitable for small animals, or they may be unstable for measurement because of external factors, including environment, light, and room temperature, and interior factors including body temperature, body gestures, etc. Furthermore, the application of these imaging methods in small animal models, especially mice, is time-intensive and requires extensive use of expensive equipment as well as expertise [12]. Some imaging methods are invasive and irreproducible [18]. This has driven the use of laser Doppler imaging and ultrasound in experimental small animal models of hindlimb ischemia.

It is generally accepted that a reliable non-invasive tool for detecting increased blood flow or perfusion after hindlimb ischemia would be highly valued in clinical practice and in preclinical research. A currently popular method is based on estimation of blood flow (ultrasonographic flow velocity waveform) or perfusion (laser Doppler) based on two legs from a single mouse, calculated blood recovery based on the ratio of blood flow (ischemic leg vs non-ischemic leg) can be then [1]. This could normalize individual variations and interference from some other factors.

One measurement based on the maximum Doppler shift waveform derived from ultrasonography is the Gosling pulsatility index (PI), a ratio of pulse amplitude to its mean value [19]. This index, being a ratio of blood velocities in one leg, is independent of vessel diameter, position, measurement angles, and temperatures [20]. Thus, data can be directly compared between mice, even from other labs. It was previously reported that PI has been successfully used to predict diseases in obstetrics, renal transplants, internal carotid, and middle cerebral arteries [21].

Herein, we describe an ultrasonographic imaging methodology that used PI values to identify blood flow recovery after hindlimb ischemia in mouse model. Compared with 


\section{Cellular Physiology Cell Physiol Biochem 2018;48:2114-2122

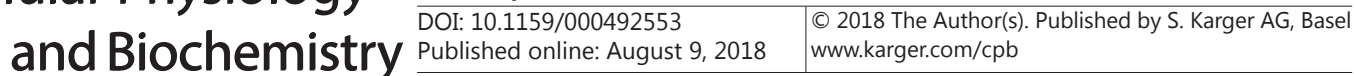 \\ Xu et al.: Pulsatility Index for Perfusion Evaluation}

non-invasive conventional techniques, the PI value generated by Doppler shift waveform showed only subtle changes of the ischemic leg in blood flow recovery following ischemic surgery. This index could also identify early differences in response to treatment of growth factor therapy. Therefore, the PI value in ischemic leg can be considered as a novel index for relatively reproducible and repeatable blood flow recovery for evaluating emerging ischemic therapies in mouse models of hindlimb ischemia.

\section{Materials and Methods}

\section{Animals}

Male C57B16/J mice were purchased from the Animal Facility at Wuhan University Zhong Nan Hospital, and then fed a standard diet with free access to water. All protocols were approved by the Institutional Animal Care and Use Committee of Yangzhou University, and were performed according with the National Institutes of Health Guide for the Care and Use of Laboratory Animals.

\section{Hindlimb ischemia mouse model}

The hindlimb ischemic model was generated as described previously. A unilateral incision was made over the left medial thigh of the mouse. During 8 to 12 weeks of age, the mice were anesthetized by intraperitoneal administration of ketamine $(30 \mathrm{mg} / \mathrm{kg})$ and xylazine $(6 \mathrm{mg} / \mathrm{kg})$. After incision of the skin in the inguinal region, the femoral artery was aseptically and carefully dissected from the vein and nerve. Two sites of the common iliac artery and the femoral artery were tightly ligated with $6 / 0$ sutures. Subsequently, the skin was closed with interrupted silk sutures. The arteries at the right side were not ligated and served as a control group.

\section{Measurement of blood flow with laser Doppler perfusion imaging (LDPI)}

Laser Doppler perfusion imaging (LDPI) was used to monitor perfusion in the ischemic and nonischemic legs before/after surgery. All the mice were evaluated under the same experimental conditions. Animals were anesthetized with $1 \%$ isoflurane and placed on a heated pad to maintain temperature at $37^{\circ} \mathrm{C}$ to minimize data variations caused by body temperature fluctuations. After anesthesia was administered, at least 10 minutes were allowed to pass to ensure a stable body temperature and to allow the mice to reach a deep anesthesia condition, as indicated by the absence of the paw reflex. To avoid any variations induced by scanning, the distance between the source of the laser and the leg was set to $21 \mathrm{~cm}$, and the resolution was $256 \times 256$ pixels. Mean perfusion was estimated in the foot and in the ischemic portion of the leg, extending from the thigh to the ankle. The non-ischemic legs and feet were used as controls. The mean perfusion results were reported as ratios of the surgery leg to non-surgery leg or foot for each animal.

\section{Measurement of blood flow with ultrasound imaging}

Blood flow in both legs was measured using a Vevo2100 high resolution micro-ultrasound system (Visualsonics Inc, Toronto, Canada) in the ischemic and non-ischemic legs before and after surgery. Mice were anesthetized using $1 \%$ isoflurane in oxygen and then fixed in the supine position on a heat pad. Electrode gel was applied to the limb leads to obtain concurrent electrocardiographic recordings during measurement [22]. The isoflurane concentration was adjusted to maintain heart rates in the physiologic range (400-500 BMP/s). Hindlimb hair was removed with depilatory cream [23]. Image acquisition was initiated with an MD550 transducer probe placed along the femoral artery to obtain the long axis view, which displays both ligation sites. Following acquisition of long-axis images at this position, the probe was moved along the femoral artery to identify the proximal caudal femoral artery (PCFA), which supplies nutrients and oxygen required in adductor muscles. Color Doppler images were captured to further confirm this artery and blood flow was examined in pulsed-wave Doppler mode. Blood flow measurements were performed under the same body conditions, except for body temperature. After anesthesia, the temperature of the animals was modified by a heated pad or by changing the isoflurane concentration. 10 minutes of equilibration time was allowed to pass in order to ensure a stable body temperature. 


\section{Cellular Physiology Cell Physiol Biochem 2018;48:2114-2122 \begin{tabular}{ll|l} 
and Biochemistry Published online: August 9, 2018 & $\begin{array}{l}\text { C } 2018 \text { The Author(s). Published by S. Karger AG, Basel } \\
\text { www.karger.com/cpb }\end{array}$ \\
\hline
\end{tabular} \\ Xu et al.: Pulsatility Index for Perfusion Evaluation}

\section{Analysis of ultrasound data}

After image acquisition, the captured images were reviewed for quality and analyzed with the Vevo working station. PI values were calculated using four continuous cardiac cycles.

$$
P I=\frac{\mathrm{Vs}-\mathrm{Vd}}{\mathrm{Va}}
$$

$\mathrm{V}_{\mathrm{s}}$ is the maximal systolic flow, $\mathrm{V}_{\mathrm{d}}$ is the end diastolic flow, and $\mathrm{V}_{\mathrm{a}}$ is the temporal average frequency over 1 cardiac cycle.

\section{Pathologic assessment of angiogenesis}

Following laser Doppler perfusion and ultrasound scanning at the 4-week time point, each animal was sacrificed. Gastrocnemius muscle was fixed in 4\% paraformaldehyde, paraffin embedded, and sectioned $(6 \mu \mathrm{m})$. The paraffin sections of the hindlimb tissue were deparaffinized using xylene (dimethyl benzene) and were heated in a citrate buffer $\left(\mathrm{pH}\right.$ 6.0) for retrieving antigen. Slides were then blocked with $3 \% \mathrm{H}_{2} \mathrm{O}_{2}$ and $10 \%$ goat serum, and were incubated with the primary antibody for vWF overnight at $4{ }^{\circ} \mathrm{C}$. The slides were then washed 3 times with PBS and incubated with a secondary for $1 \mathrm{~h}$ at $37^{\circ} \mathrm{C}$. DAPI was used to counterstain the nuclei and then washed. Replacement of the primary antibody with PBS served as a negative control. Sections were imaged with a microscope using a $20 \mathrm{X}$ objective. The number of animal microvessels per group and eight fields of view per tissue were counted. All measurements were performed in a blinded manner [24].

\section{Statistical analysis}

All data were shown as means \pm SD. Statistical analysis was carried out using SPSS Version 23. A $t$-test was used to compare the means between two groups. A two-way analysis of variance (ANOVA) was used to compare measurements of various parameters over time.

\section{Results}

PI precedes blood recovery in experimental hindlimb ischemia

We investigated the temporal relationship between PI and blood flow recovery in the hindlimb ischemia mouse model. First, the blood flow increase in the hindlimb ischemic mouse model was measured with a laser Doppler perfusion imaging system. One day after ligation surgery, the ratio of perfusion in the ischemic leg to that in the non-ischemic leg sharply decreased to $8.0 \pm 1.6 \%$ compared to the ratio before ligation (Fig. 1A). With an endogenous repairing system, the perfusion ratio increased to $12.3 \pm 2.8 \%$ two weeks after ligation and reached $17.1 \pm 1.9 \%$ four weeks after surgery (Fig. 1B).

The velocity of blood flow in the proximal caudal femoral artery was measured with a micro-ultrasound system before and after surgery at different times. To normalize the difference between two legs, the ratio of blood flow was calculated as $101.1 \pm 7.5 \%$ in the normal condition. In this way, the ratio of blood flow significantly decreased to $6.6 \pm 1.4 \%$ after ligation compared with that before surgery (Fig. 1B). With the endogenous angiogenic repairing system, the blood flow ratio reached $11.4 \pm 2.8 \%$ four weeks after surgery (Fig. 1B).

Besides the consistency of the perfusion ratio (ligation leg vs non-ischemic leg) from laser Doppler perfusion imaging and the blood flow ratio measured by ultrasound, both methods also displayed endogenous recovery of blood flow after ligation. The ratio of perfusion was positively correlated with the blood flow ratio measured by ultrasound (Fig. 1D-1F).

Blood flow analysis was performed in pulsed-wave Doppler images, and the peak systolic velocity $\left(V_{\mathrm{s}}\right)$, minimal end diastolic velocity $\left(V_{\mathrm{d}}\right)$, and average velocity per heart cycle $\left(\mathrm{V}_{\mathrm{a}}\right)$ in the femoral arteries were acquired (Fig. 2A). Temporal analysis of PI was performed in the proximal caudal femoral artery, which was downstream of the ligation sites and primarily supplied blood to the adduct muscle. We found a drastic decrease of PI after surgery; the value reached 0 compared to the value before ischemic surgery (Fig. $2 \mathrm{~B}$ and $2 \mathrm{C}$ ). Then, there 
Fig. 1. The mouse model of hindlimb ischemia. A. The representative images for perfusion measured by laser Doppler system before and 28 days after surgery. B. The ratio for perfusion measured by laser Doppler system at the different time points in mouse model of hindlimb ischemia. C. The ratio for blood flow measured by microultrasound at the different time points in mouse model of hindlimb ischemia. D. The correlation between the ratios for perfusion measured by laser Doppler system and blood flow measured by micro-ultrasound 14 days after surgery. E. The correlation between the ratios for perfusion measured by laser Doppler system and blood flow measured by microultrasound 21 days after surgery. F. The correlation between the ratios for perfusion measured by laser Doppler system and blood flow measured by micro-ultrasound 28 days after surgery. $\mathrm{n}=8$ each group, ${ }^{*} \mathrm{p}<0.05$ vs. 0 day.

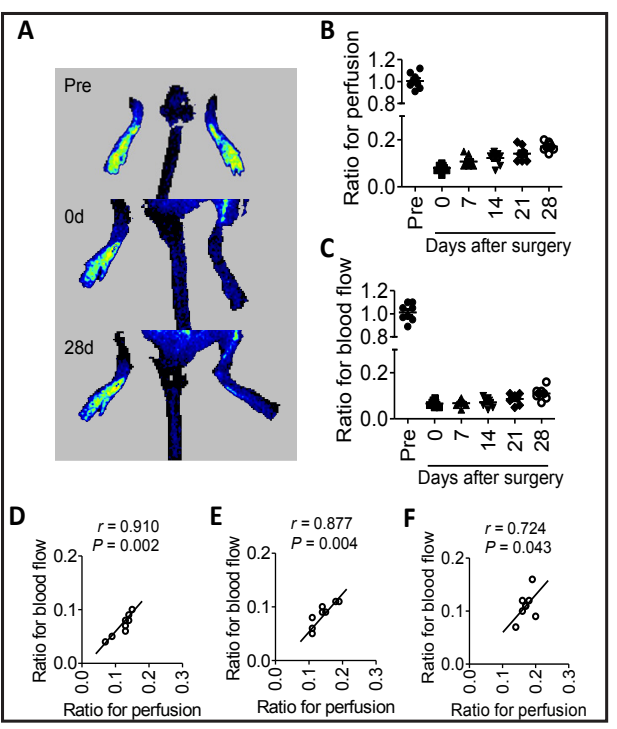

was a continuous increase in PI after ligation (Fig. $2 \mathrm{~B}$ and $2 \mathrm{C}$ ).

\section{PI correlation with blood recovery in hindlimb ischemia \\ To further investigate PI as an index for} blood flow recovery in hindlimb ischemia, the correlation between PI and the blood flow ratio measured with other methods was studied in the mouse model of ischemic hindlimb. 28 days after ligation surgery, there was a strong linear relationship between PI and perfusion ratio measured with a laser Doppler perfusion image $(r=0.915,95 \%$ CI, 0.591 to $0.985, p=0.002$ ) (Fig. 2D). The correlation between PI and blood flow ratio was also identified from ultrasound $(r=$ $0.893,95 \%$ CI, 0.508 to $0.981, p=0.003$ ) (Fig. 2E).

Taken together, these data suggest that PI in the proximal caudal femoral artery could be used to evaluate blood flow recovery in the mouse model of hindlimb ischemia.

\section{PI correlation with angiogenesis in hindlimb ischemia}

Using detection of PI temporal changes in the proximal caudal femoral artery in hindlimb ischemia, we further investigated the relationship between PI and angiogenesis after ligation.

vWF, a molecular marker for endothelial cells, did not significantly increase in the ischemic leg 7 days after ligation compared to the sham leg (Fig. 3A-3C). However, positive signaling of vWF was significantly increased in the ischemic leg 28 days after ligation (Fig. 3A-3C).

Then, the relationship between PI and angiogenesis was analyzed at different blood flow recovery stages after ligation. PI was correlated with vessel number 7 days after ligation 


\section{Cellular Physiology Cell Physiol Biochem 2018:48:2114-2122 \begin{tabular}{l|l|l} 
DOI: 10.1159/000492553 & and Biochemistry & $\begin{array}{l}\text { O } 2018 \text { The Author(s). Published by S. Karger AG, Basel } \\
\text { www.karger.com/cpb }\end{array}$
\end{tabular} Xu et al.: Pulsatility Index for Perfusion Evaluation}

( $r=0.919,95 \% \mathrm{CI}, 0.607$ to $0.985, p=0.001$ ) (Fig. 3D), and the relationship became closer 28 days after surgery ( $r=0.988,95 \% \mathrm{CI}, 0.5931$ to $0.998, p$ $<0.001$ ) (Fig. 3E).

The ability of PI measures to differentiate effects of ischemic treatment

To determine the relative sensitivity of PI analyses versus conventional blood flow ratios measured by laser Doppler imaging during blood flow recovery, mice were treated with FGF2 or PBS (control) after hindlimb ischemia. Both conventional and PI analyses showed similar results at basic conditions, before or after surgery. PI analysis showed that FGF2 significantly promoted blow flow compared with PBS treatment (twoway ANOVA analysis, $\mathrm{p}<0.05$ ) (Fig. 4A and 4B). In mice injected with FGF2, the PI value increased significantly two weeks after treatment compared to the control group (Fig. 4A and 4B). Four weeks after treatment, PI value was approximately a factor 1.968 larger in mice treated with FGF2 than those treated with PBS (Fig. 4A and 4B).

In mice injected with FGF2, the blood flow ratio measured by laser Doppler perfusion imaging significantly increased two weeks after treatment compared to the control mice (Fig. 4C). Four weeks after treatment, the blood flow ratio of the FGF2 treatment group was approximately a factor 2.767 larger than PBS control (Fig. 4C). In addition, the significantly increased blood flow ratio measured by ultrasound was identified in the FGF2 administrated mice 2 weeks after treatment (Fig. 4D). Consistent with previous reports, laser Doppler perfusion and ultrasound analysis showed that FGF2 can significantly promote perfusion and blood flow compared with PBS (two-way ANOVA analysis).

\section{Discussion}

The aim of this study was to establish and evaluate a non-invasive ultrasonographic imaging methodology for PI, thus revealing blood flow recovery after hindlimb ischemia in mouse model. The PI value in the ischemic leg could be considered a novel index for relatively reproducible and repeatable blood flow recovery, including the evaluation of emerging ischemic therapies in mouse model of hindlimb ischemia.

Currently, there are some invasive perfusion methods for preclinical research with a contrast agent in the application of CT, MRI, and other techniques. The contrast agent in MRI offers relative good sensitivity and spatial resolution in vascular imaging, in which the circulatory system including capillary vessel can be identified and separated from the background tissue. To obtain higher spatial resolution images with MRI, the mice must be sacrificed to allow a sufficient frame rate for bolus tracking [25]. Although contrast agents highly increase the sensitivity for scanning, SPECT and PET both suffered from poor spatial resolution. Even though these techniques can provide good images for analysis of perfusion, they require expensive equipment and operational expertise. Moreover, injection 


\section{Cellular Physiology Cell Physiol Biochem 2018;48:2114-2122

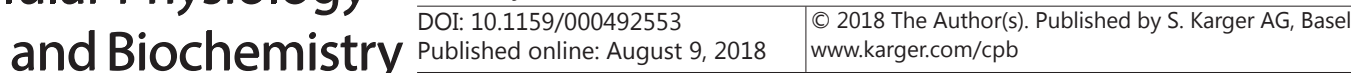 Xu et al.: Pulsatility Index for Perfusion Evaluation}

of contrast agents for commonly used black mice with C57B16/J background could be challenging and time consuming.

Recently, other approaches to noninvasive perfusion assessment, including laser Doppler and some ultrasound techniques, have been explored to provide simultaneous imaging of perfusion and blood flow. Although the current golden standard for reperfusion measurement is to use laser Doppler in preclinical research on reperfusion in mouse model of hindlimb ischemia, it still has many disadvantages. The perfusion value with laser Doppler can be disturbed by external environmental factors, including light intensity, environmental temperature, and temperature fluctuations. Additionally, some internal factors also can affect the measurement accuracy and stability. The perfusion value from laser Doppler correlates well with mouse body temperatures. Moreover, some details in the measurement procedure can determine the perfusion value, resulting in repeatability and data comparability. It is necessary to maintain the same imaging position in order to maintain the same distances between the scanner and both sides of the feet/ legs in one mouse. Tiny fluctuations in anesthetic conditions can change the perfusion value. To avoid some of the disadvantages of laser Doppler, the blood blow ratio from two sides of a mouse is adapted in analysis of perfusion. However, the mouse position and distances between the scanner and feet/legs are not fixed in real-time

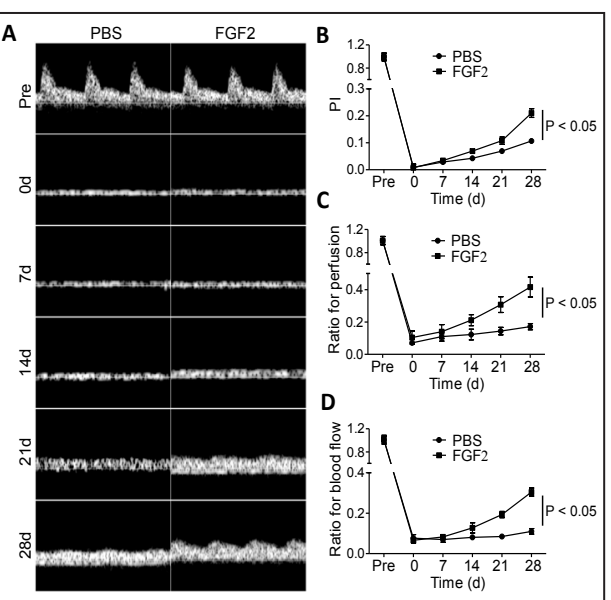

Fig. 4. PI could be used to monitor the perfusion induced by FGF2. A. The representative $\mathrm{PW}$-model images of the femoral arteries. Blood flow was measured at the different time points after ischemia with PBS or FGF2. B. The PI values were showed at the different time points after surgery in mouse model of hindlimb ischemia with PBS or FGF2. C. The ratios for perfusion measured by laser Doppler system were showed at the different time points after surgery in mouse model of hindlimb ischemia with PBS or FGF2. D. The ratios for blood flow measured by micro-ultrasound were showed at the different time points after surgery in mouse model of hindlimb ischemia with PBS or FGF2. $\mathrm{n}=8$ each group, ${ }^{*} \mathrm{p}<0.05$ vs. PBS. measurements for an extended period of time.

Normal ultrasonography for blood flow is involved in a typical pattern of color Doppler and pulsed-wave velocity mode imaging. Like laser Doppler, it depends on the blood flow velocity measurement of both legs for a single mouse. There are also many disadvantages of blood flow measurement with ultrasound compared to laser Doppler. The measurement angle is another drawback for blood flow analysis using ultrasound. A small change of angle sharply affects the measured peak velocity of blood flow and fails to reflect the tiny change of blood flow in the recovery process after ischemia. Even an analysis of blood flow ratio between the two sides of feet/legs cannot overcome this disadvantage.

Accordingly, we observed alterations in the PI value, which tended to be much easier and more sensitive in preclinical research of ischemic mice. We adopted the PI value as a new index for interrupting collateral flow and blood flow recovery. With this method, the blood flow recovery in distal hindlimb tissue could be consistently measured, which tended to give a more accurately representation of angiogenesis and arteriogenesis. Because the PI value is a ratio of pulse amplitude to its mean value, as a ratio of velocities in one leg, it did not rely on vessel diameter information, position, measurement angles, or external and internal temperatures. Importantly, it was not affected by the mouse position and the distance between the scanner and legs. Therefore, this analysis of blood flow recovery was more repeatable and reproducible. Using PI analysis, the blood flow increase could be easily estimated in only one ischemic leg during the course of recovery following ischemia. Thus, it was efficient to monitor the blood flow from only one ischemic leg, rather than from both legs with laser Doppler. 


\section{Cellular Physiology Cell Physiol Biochem 2018;48:2114-2122 \begin{tabular}{l|l} 
and Biochemistry Published 10.1159/000492553 & $\begin{array}{l}\text { (c) 2018 The Author(s). Published by S. Karger AG, Basel } \\
\text { www.karger.com/cpb }\end{array}$ \\
\hline
\end{tabular} \\ Xu et al.: Pulsatility Index for Perfusion Evaluation}

Our results also demonstrate that serial application of PI analysis from blood flow measurements in the ischemic leg is highly convenient and allows one to easily identify any beneficial effects of an angiogenic therapy following ischemia. In contrast, conventional perfusion and flow measurements with laser Doppler or ultrasound could not detect the difference between PBS versus FGF-2-treated mice until 2 weeks following ischemia (Fig. 4C and 4D). Prior studies have also shown significantly improved blood flow 2 weeks after FGF2 treatment. Our data indicated that PI value alterations can directly reflect the effects of rescue therapy with FGF- 2 treatment 2 weeks after injection and also predicted subsequent differences in blood flow recovery (Fig. 4B).

The limitations for this study merit consideration. In this study, only an acute ischemia mouse model was examined. Multiple models should be validated in future studies. Another issue was that further clinical evidence is required due to human disease complications, such as atherosclerosis, diabetes, and other diseases.

In conclusion, the present study introduces a novel noninvasive imaging analysis for assessing blood flow recovery after ligation in the hindlimb of a mouse. Importantly, given the affect from many aspects in experimental procedures, this methodology is a potential candidate for rapid estimation in a relatively large number of animals over a longer experimental time. Furthermore, PI value analysis allows for rapid and sensitive assessment of blood flow recovery in response to either emerging therapies after hindlimb ischemia or in response to potential therapeutic agents. This technique is particularly useful in the growing availability of transgenic mice.

\section{Acknowledgements}

This study was supported by a grant from the National Natural Science Foundation of China (No. 81500720, 81303109), a grant from the Health Bureau Technology Fund of Tianjin (No. 2014KZ092), the Research Fund for the Doctoral Program of Higher Education of China (20113250120006), and the Natural Science Fund of Yangzhou City (YZ2014047).

\section{Disclosure Statement}

No conflict of interests exists.

\section{References}

1 Lu Q, Yao Y, Yao Y, Liu S, Huang Y, Lu S, Bai Y, Zhou B, Xu Y, Li L, Wang N, Wang L, Zhang J, Cheng X, Qin G, Ma W, Xu C, Tu X, Wang Q: Angiogenic factor AGGF1 promotes therapeutic angiogenesis in a mouse limb ischemia model. PLoS One 2012;7:e46998.

$\checkmark 2$ Hiatt WR: Medical treatment of peripheral arterial disease and claudication. N Engl J Med 2001;344:16081621.

- Lange S, Diehm C, Darius H, Haberl R, Allenberg JR, Pittrow D, Schuster A, von Stritzky B, Tepohl G, Trampisch HJ: High prevalence of peripheral arterial disease but low antiplatelet treatment rates in elderly primary care patients with diabetes. Diabetes Care 2003;26:3357-3358.

-4 Rooke TW, Hirsch AT, Misra S, Sidawy AN, Beckman JA, Findeiss LK, Golzarian J, Gornik HL, Halperin JL, Jaff MR, Moneta GL, Olin JW, Stanley JC, White CJ, White JV, Zierler RE, Society for Cardiovascular A, Interventions, Society of Interventional R, Society for Vascular M, Society for Vascular S: 2011 ACCF/ AHA Focused Update of the Guideline for the Management of Patients With Peripheral Artery Disease (updating the 2005 guideline): a report of the American College of Cardiology Foundation/American Heart Association Task Force on Practice Guidelines. J Am Coll Cardiol 2011;58:2020-2045. 


\section{Cellular Physiology Cell Physiol Biochem 2018;48:2114-2122 \begin{tabular}{ll|l} 
and BiOChemistry & $\begin{array}{l}\text { DOI: 10.1159/000492553 } \\
\text { Published online: August 9, } 2018\end{array}$ & $\begin{array}{l}\text { (c) } 2018 \text { The Author(s). Published by S. Karger AG, Basel } \\
\text { www.karger.com/cpb }\end{array}$
\end{tabular} \\ Xu et al.: Pulsatility Index for Perfusion Evaluation}

5 Marso SP, Hiatt WR: Peripheral arterial disease in patients with diabetes. J Am Coll Cardiol 2006;47:921929.

6 Criqui MH, Langer RD, Fronek A, Feigelson HS, Klauber MR, McCann TJ, Browner D: Mortality over a period of 10 years in patients with peripheral arterial disease. N Engl J Med 1992;326:381-386.

-7 Dieter RS, Tomasson J, Gudjonsson T, Brown RL, Vitcenda M, Einerson J, McBride PE: Lower extremity peripheral arterial disease in hospitalized patients with coronary artery disease. Vasc Med 2003;8:233236.

-8 Welten GM, Schouten 0, Chonchol M, Hoeks SE, Bax JJ, Van Domburg RT, Poldermans D: Prognosis of patients with peripheral arterial disease. J Cardiovasc Surg (Torino) 2009;50:109-121.

-9 Xing J, Li J: Proteinase-Activated Receptor-2 Sensitivity of Amplified TRPA1 Activity in Skeletal Muscle Afferent Nerves and Exercise Pressor Reflex in Rats with Femoral Artery Occlusion. Cell Physiol Biochem 2017;44:163-171.

10 Xing J, Li J: TRPA1 Function in Skeletal Muscle Sensory Neurons Following Femoral Artery Occlusion. Cell Physiol Biochem 2017;42:2307-2317.

11 Lu Q Xie Z, Yan C, Ding Y, Ma Z, Wu S, Qiu Y, Cossette SM, Bordas M, Ramchandran R, Zou MH: SNRK (Sucrose Nonfermenting 1-Related Kinase) Promotes Angiogenesis In vivo. Arterioscler Thromb Vasc Biol 2018;38:373-385.

12 Xu H, Ma Z, Lu S, Li R, Lyu L, Ding L, Lu Q: Renal Resistive Index as a Novel Indicator for Renal Complications in High-Fat Diet-Fed Mice. Kidney Blood Press Res 2017;42:1128-1140.

13 Lu Q Jiang X, Zhang C, Zhang W, Zhang W: Noninvasive Regional Aortic Stiffness for Monitoring the Early Stage of Abdominal Aortic Aneurysm in Mice. Heart Lung Circ 2017;26:395-403.

14 Steventon JJ, Trueman RC, Ma D, Yhnell E, Bayram-Weston Z, Modat M, Cardoso J, Ourselin S, Lythgoe M, Stewart A, Rosser AE, Jones DK: Longitudinal in vivo MRI in a Huntington's disease mouse model: Global atrophy in the absence of white matter microstructural damage. Sci Rep 2016;6:32423.

-15 Starosolski Z, Villamizar CA, Rendon D, Paldino MJ, Milewicz DM, Ghaghada KB, Annapragada AV: Ultra High-Resolution In vivo Computed Tomography Imaging of Mouse Cerebrovasculature Using a Long Circulating Blood Pool Contrast Agent. Sci Rep 2015;5:10178.

16 Zagorchev L, Mulligan-Kehoe MJ: Molecular imaging of vessels in mouse models of disease. Eur J Radiol 2009;70:305-311.

17 Ropinski T, Hermann S, Reich R, Schafers M, Hinrichs K: Multimodal vessel visualization of mouse aorta PET/CT scans. IEEE Trans Vis Comput Graph 2009;15:1515-1522.

18 Roche B, Vanden-Bossche A, Normand M, Malaval L, Vico L, Lafage-Proust MH: Validated Laser Doppler protocol for measurement of mouse bone blood perfusion - response to age or ovariectomy differs with genetic background. Bone 2013;55:418-426.

19 Michel E, Zernikow B: Gosling's Doppler pulsatility index revisited. Ultrasound Med Biol 1998;24:597-599.

20 Zarrinkoob L, Ambarki K, Wahlin A, Birgander R, Carlberg B, Eklund A, Malm J: Aging alters the dampening of pulsatile blood flow in cerebral arteries. J Cereb Blood Flow Metab 2016;36:1519-1527.

-21 Perko MJ: Duplex ultrasound for assessment of superior mesenteric artery blood flow. Eur J Vasc Endovasc Surg 2001;21:106-117.

22 Zhou Y, Xiao H, Wu J, Zha L, Zhou M, Li Q, Wang M, Shi S, Li Y, Lyu L, Wang Q, Tu X, Lu Q: Type I Diabetic Akita Mouse Model is Characterized by Abnormal Cardiac Deformation During Early Stages of Diabetic Cardiomyopathy with Speckle-Tracking Based Strain Imaging. Cell Physiol Biochem 2018;45:1541-1550.

23 Yao YF, Lu QL, Hu ZK, Yu YB, Chen QY, Wang QK: A non-canonical pathway regulates ER stress signaling and blocks ER stress-induced apoptosis and heart failure. Nat Commun 2017;8:133.

24 Ma Z, Zhu L, Liu Y, Wang Z, Yang Y, Chen L, Lu Q: Lovastatin Alleviates Endothelial-to-Mesenchymal Transition in Glomeruli via Suppression of Oxidative Stress and TGF-beta1 Signaling. Front Pharmacol 2017;8:473.

25 Rissanen TT, Korpisalo P, Markkanen JE, Liimatainen T, Orden MR, Kholova I, de Goede A, Heikura T, Grohn $\mathrm{OH}$, Yla-Herttuala S: Blood flow remodels growing vasculature during vascular endothelial growth factor gene therapy and determines between capillary arterialization and sprouting angiogenesis. Circulation 2005;112:3937-3946. 